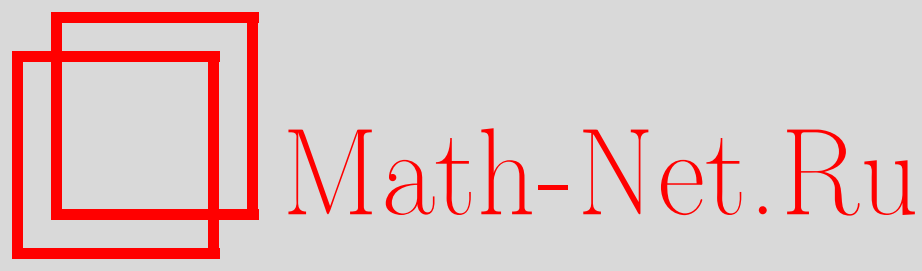

В. П. Маслов, О сверхтекучести классической жидкости в нанотрубке для четного и нечетного числа нейтронов в молекуле, ТМФ, 2007, том 153, номер 3, 388-408

DOI: https://doi.org/10.4213/tmf6144

Использование Общероссийского математического портала Math-Net.Ru подразумевает, что вы прочитали и согласны с пользовательским соглашением http://www.mathnet.ru/rus/agreement

Параметры загрузки :

IP : 35.173 .137 .237

26 апреля 2023 г., 13:30:04

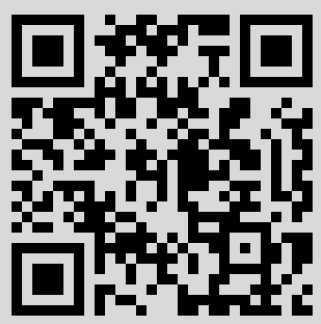




\section{О СВЕРХТЕКУЧЕСТИ КЛАССИЧЕСКОЙ ЖИДКОСТИ В НАНОТРУБКЕ ДЛЯ ЧЕТНОГО И НЕЧЕТНОГО ЧИСЛА НЕЙТРОНОВ В МОЛЕКУЛЕ}

Показано, что в нанотрубках возникает явление сверхтекучести для классических жидкостей.

Ключевые слова: сверхтекучесть, бозоны, фермионы.

\section{1. ВВЕДЕНИЕ}

1.1. Случай четного числа нейтронов. Отметим прежде всего, что решения уравнения в вариациях для уравнения Власова не совпадают с классическим пределом для уравнений в вариациях для уравнений среднего поля в квантовой теории.

Рассмотрим уравнения среднего поля вида

$$
i h \frac{\partial}{\partial t} \varphi^{t}(x)=\left(-\frac{h^{2}}{2 m} \Delta+W_{t}(x)\right) \varphi^{t}(x), \quad W_{t}(x)=U(x)+\int d y V(x, y)\left|\varphi^{t}(y)\right|^{2}
$$

при начальном условии $\left.\varphi\right|_{t=0}=\varphi_{0}$, где $\varphi_{0} \in W_{2}^{\infty}\left(\mathbb{R}^{\nu}\right), \quad \int d x\left|\varphi_{0}(x)\right|^{2}=1$.

Для получения асимптотики типа комплексного ростка [1] рассмотрим систему, состоящую из уравнения Хартри и уравнения, ему сопряженного. Затем рассмотрим для нее систему в вариациях и, наконец, заменим вариации $\delta \varphi$ и $\delta \varphi^{*}$ на независимые функции $F$ и $G$. Для функций $F$ и $G$ получаем следующую систему уравнений:

$$
\begin{aligned}
i \frac{\partial F^{t}(x)}{\partial t} & =\int d y\left(\frac{\delta^{2} H}{\delta \varphi^{*}(x) \delta \varphi(y)} F^{t}(y)+\frac{\delta^{2} H}{\delta \varphi^{*}(x) \delta^{*} \varphi(y)} G^{t}(y)\right), \\
-i \frac{\partial G^{t}(x)}{\partial t} & =\int d y\left(\frac{\delta^{2} H}{\delta \varphi(x) \delta \varphi(y)} F^{t}(y)+\frac{\delta^{2} H}{\delta \varphi(x) \delta^{*} \varphi(y)} G^{t}(y)\right) .
\end{aligned}
$$

Классические уравнения получаются из квантовых, грубо говоря, путем подстановки вида $\varphi=\chi e^{i S / h}$ (метод ВКБ), $\varphi^{*}=\chi^{*} e^{-i S^{*} / h}, \quad S=S^{*}, \quad \chi=\chi(x, t) \in C^{\infty}$, $S=S(x, t) \in C^{\infty}$.

* Московский государственный университет, Москва, Россия. E-mail: v.p.maslov@mail.ru 
Для уравнения в вариациях естественно варьировать не только предельное уравнение для $\chi$ и $\chi^{*}$, но и функции $S$ и $S^{*}$. Это дает новый важный член решения уравнения для коллективных колебаний.

Проследим этот факт на простейшем примере, который исследуется в знаменитой работе Боголюбова о слабонеидеальном бозе-газе [2].

Пусть в уравнении (1) $U=0$ в трехмерном ящике с длиной ребра $L$, при этом на волновые функции наложено условие периодичности (т.е. речь идет о задаче на торе с образующими $L, L, L)$. Тогда функция

$$
\varphi(x)=L^{-3 / 2} e^{\frac{i}{h}(p x-\Omega t)},
$$

где $p=2 \pi n / L, n$ - целочисленный вектор, удовлетворяет уравнению (1) при

$$
\Omega=\frac{p^{2}}{2 m}+L^{-3} \int d x V(x) .
$$

Рассмотрим функции $F^{(\lambda)}(x)$ и $G^{(\lambda)}(x)$, где $\lambda=2 \pi n / L, n \neq 0$, следующего вида:

$$
\begin{aligned}
& F^{(\lambda) t}(x)=L^{-3 / 2} \rho_{\lambda} e^{\frac{i}{h}|(p+\lambda) x+(\beta-\Omega) t|}, \\
& G^{(\lambda) t}(x)=L^{-3 / 2} \sigma_{\lambda} e^{\frac{i}{h}|(-p+\lambda) x+(\beta+\Omega) t|},
\end{aligned}
$$

где

$$
\begin{gathered}
-\beta_{\lambda} \rho_{\lambda}=\left(\frac{(p+\lambda)^{2}}{2 m}-\frac{p^{2}}{2 m}+\widetilde{V}_{\lambda}\right) \rho_{\lambda}+\widetilde{V}_{\lambda} \sigma_{\lambda} \\
\beta_{\lambda} \rho_{\lambda}=\left(\frac{(p-\lambda)^{2}}{2 m}-\frac{p^{2}}{2 m}+\widetilde{V}_{\lambda}\right) \sigma_{\lambda}+\widetilde{V}_{\lambda} \rho_{\lambda} \\
\left|\sigma_{\lambda}\right|^{2}-\left|\rho_{\lambda}\right|^{2}=1, \quad \widetilde{V}_{\lambda}=L^{-3} \int d x V(x) e^{\frac{i}{h} \lambda x}
\end{gathered}
$$

Из системы (6) находим

$$
\beta_{\lambda}=-p \lambda+\sqrt{\left(\frac{\lambda^{2}}{2 m}+\widetilde{V}_{\lambda}\right)^{2}-\widetilde{V}_{\lambda}^{2}}
$$

В этом примере $u=e^{\frac{i}{h} s(x, t)}, u^{*}=e^{-\frac{i}{h} s(x, t)}$, где $s(x, t)=,p x+\beta t$, а вариация действия для вектора $\left(\delta u, \delta u^{*}\right)$ равна $\lambda x \pm \Omega t$. При более тщательном переходе к пределу $\widetilde{V}_{\lambda} \rightarrow V_{0}=L^{-3} \int d x V(x)$.

Таким образом, в классическом пределе мы получаем знаменитое соотношение Боголюбова (7). В этом случае $u(x)=0$, как и в линейном уравнении Шредингера, точное решение совпадает с квазиклассическим. В работе [3] исследуется случай $u(x) \neq 0$, и оказывается, что соотношение, аналогичное $(7)$, является классическим пределом при $h \rightarrow 0$ для уравнения в вариациях в этом общем случае. Кривая зависимости $\beta_{\lambda}$ от $\lambda$ называется кривой Ландау и определяет сверхтекучее состояние. Значение $\lambda_{\text {кр }}$, при котором сверхтекучесть пропадает, называется критерием Ландау. Боголюбов объясняет явление сверхтекучести следующим образом: «"върожденньй конденсат" может двигаться без трения относительно элементарных возбуждений с произвольной достаточно малой скоростью» ([2], с. 210). 
Однако о бозе-эйнштейновском конденсате в этом математическом рассмотрении

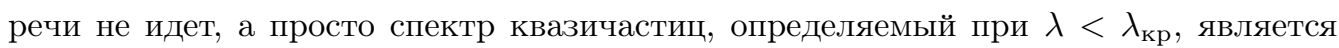
положительным. Это означает его метастабильность (см. [4]). Бозе-эйнштейновский конденсат здесь упоминается лишь для того, чтобы в силу вышеизложенного не возникала мысль, что это рассуждение подходит для классической жидкости.

Действительно, например, молекулы классической неразряженной жидкости являются бозе-частицами, если число нейтронов в молекуле четно. Для такой жидкости можно написать $N$-частичное уравнение, имея в виду, что каждая частица (молекула) нейтральна и состоит из четного числа $l$ нейтронов. Таким образом, каждая $i$-я частица $3(2 k+l)$-мерна, где $k$ - число электронов, имеется зависимость от потенциала $u\left(x_{i}\right), x_{i} \in \mathbb{R}^{6 k+3 l}$, и можно рассмотреть уравнение для $N$ частиц $x_{i}$, $i=1,2, \ldots, N$, с парным взаимодействием $V\left(x_{i}-x_{j}\right)$.

Однако Боголюбов нашел лишь одну серию спектра многочастичной задачи. Ландау писал: "Н.Н. Боголюбову недавно удалось с помощью остроумного применения вторичного квантования определить в общем виде энергетический спектр бозе-эйнштейновского газа со слабым взаимодействием между частицами" ([5], с. 43). Но эта серия не является единственной, т.е. она не исчерпывает весь энергетический спектр.

Автор в 2001 г. предложил метод ультравторичного квантования ([6]; см. также [7]-[11]). Ультравторично-квантованные уравнения Шредингера, как и вторичноквантованные, являются некоторым представлением $N$-частичного уравнения Шредингера, а это значит, что, по существу, ультравторично-квантованное уравнение тождественно исходному $N$-частичному уравнению: совпадает с ним на $3 N$-мерном пространстве. Однако замена операторов рождения и уничтожения $c$-числами, в отличие от вторично-квантованного случая, еще не дает правильной асимптотики, но оказывается, она совпадает с результатом применения вариационного принципа Шредера или вариационного метода Боголюбова.

Для экзотического потенциала Бардина правильное асимптотическое решение совпадает с тем, которое получается в результате применения вышеуказанного метода ультравторичного квантования. Для потенциалов общего вида, например для парного взаимодействия, ответ оказывается другой. В частности, метод ультравторичного квантования дает другие асимптотические серии собственных значений, отвечающих $N$-частичному уравнению Шредингера, которые, в отличие от боголюбовской серии (7), не являются метастабильными. Они отвечают вихревым нитям [12].

Оказывается, дело не в бозе-эйнштейновском конденсате, а в толщине капилляра (нанотрубки), по которому течет жидкость. Если мы рассматриваем жидкость в капилляре или нанотрубке, то при достаточно малом их радиусе скорость, отвечающая метастабильным состояниям, оказывается не малой. Значит, с меньшей скоростью течение будет происходить без трения.

Условие непротекания через границу нанотрубки (отсутствие потока) есть краевое условие Дирихле или краевое условие Борна-фон Кармана. Оно порождает 
стоячую волну, которую можно трактовать как пару частица-античастица: частица с импульсом $p$, ортогональным стенке трубки, и античастица с импульсом $-p$.

В бозонном случае мы рассматриваем короткодействующий потенциал взаимодействия $V\left(x_{i}-x_{j}\right)$. Это означает, что при числе частиц $N \rightarrow \infty$ взаимодействие возможно лишь с конечным числом частиц. А значит, потенциал зависит от $N$ следующим образом: $V_{N}=V\left(\left(x_{i}-x_{j}\right) N^{1 / 3}\right)$. Если $V(y)$ финитно с носителем $\Omega_{V}$, то при $N \rightarrow \infty$ носитель захватывает конечное число частиц, не зависящее от $N$. В результате оказывается, что при скоростях, меньших $\min \left(\lambda_{\text {кр }}, h /(2 m R)\right)$, где $R-$ радиус нанотрубки, имеет место сверхтекучесть. Ограничение сверху определяется условием, что радиус действия молекулы должен быть меньше, чем радиус нанотрубки.

Теперь приведу мои собственные соображения, не имеющие отношения к математическому изложению. Вязкость связана со столкновением частиц: чем больше температура, тем больше столкновений. В нанотрубке столкновений мало, происходят только столкновения со стенками, что учитывается полученной ниже серией. Именно это обстоятельство, а не наличие бозе-конденсата, приводит к ослаблению вязкости и, следовательно, к сверхтекучести. То есть основным фактором явления сверхтекучести даже жидкого $\mathrm{He}^{4}$ является не конденсат, а наличие тонкого капилляра [13], [14].

1.2. Случай нечетного числа нейтронов. В этом случае важна не отталкивающая часть потенциала, а его притягивающая часть, и, значит, необходимо более точное знание поведения потенциала на бесконечности.

Для рассмотрения системы, в которой число нейтронов в молекуле нечетно, а значит, спин ее равен $1 / 2$, что приводит к антисимметрии собственных функций, мы используем потенциал взаимодействия, который обеспечивает отталкивание на близких расстояниях и притяжение на далеких расстояниях. Таким свойством обладает, в частности, потенциал взаимодействия в ферми-жидкости $\mathrm{He}^{3}$.

Потенциал $V(\xi)$ будем считать сферически-симметричным. Отсюда следует, что и его фурье-образ $\widetilde{V}(\xi)$ будет также сферически-симметричным. Далее мы поставим чисто математическое условие, которое назовем свойством зеркальности. Как потенциал $V(\xi)$, так и его образ $\widetilde{V}(\xi)$ должны иметь ветвление вида $\sqrt{|x|}=\sqrt{x_{1}^{2}+x_{2}^{2}+x_{3}^{2}}$. Возможно даже, что это условие является лишним для приведенных ниже результатов, но оно возникает в целом ряде других задач, и в нем заложен глубокий смысл при аналитическом продолжении (см., например, [15]). Это означает, что $V(r)$ существенно зависит от первой степени радиуса $r$, и его фурье-образ обладает тем же свойством относительно $|p|$.

Переход к сферическим координатам приводит к соотношению1)

$$
V(r)=\frac{4 \pi}{r} \int_{0}^{\infty} \tilde{V}(\xi) \sin (\xi r) \xi d \xi
$$

1)Эту формулу мне сообщил М. В. Карасёв. 
В предположении, что $\widetilde{V}(|\xi|)$ стремится к бесконечности как const $/|\xi|^{2}$, мы получим, что

$$
V(r)=\frac{\text { const }}{r}+O(1)
$$

при $r \rightarrow 0$ и $V(r) \rightarrow \infty$ как const $/ r^{4}$.

Условие зеркальности подразумевает, что $\left.\widetilde{V}_{|\xi|}^{\prime}(|\xi|)\right|_{|\xi|=0} \neq 0$. Нетрудно убедиться, что

$$
\left.\widetilde{V}_{|\xi|}\right|_{|\xi|=0}=-\frac{1}{8 \pi} \lim _{r \rightarrow \infty} r^{4} V(r) .
$$

Известно, что в свободном пространстве потенциал взаимодействия убывает как $r^{-6}$, однако если добавить произвольно малый радиально-симметричный потенциал, то почти наверняка убывание будет иметь порядок $r^{-4}$.

\section{2. УЛЬТРАВТОРИЧНОЕ КВАНТОВАНИЕ}

В работах [6], [16]-[18] было введено ультравторичное квантование для задач квантовой механики и статистической физики. Напомним обозначения и основные факты в случае квантования по парам частица-номер частицы и по парам из двух частиц. Квантование с учетом пар позволит далее учесть парные корреляции частиц при построении асимптотики. Пространством ультравторичного квантования является бозонное пространство Фока $\mathcal{F}, \hat{b}^{+}(x, s)$ - оператор рождения частиц с номером $s, \hat{b}^{-}(x, s)$ - оператор уничтожения частиц с номером $s$ в пространстве $\mathcal{F}$ [19], $\widehat{B}^{+}\left(x, x^{\prime}\right)$ - оператор рождения пары частиц, $\widehat{B}^{-}\left(x, x^{\prime}\right)$ - оператор уничтожения пары частиц в этом пространстве. Эти операторы удовлетворяют коммутационным соотношениям

$$
\begin{gathered}
{\left[\hat{b}^{-}(x, s), \hat{b}^{+}\left(x^{\prime}, s^{\prime}\right)\right]=\delta_{s s^{\prime}} \delta\left(x-x^{\prime}\right), \quad\left[\hat{b}^{ \pm}(x, s), \hat{b}^{ \pm}\left(x^{\prime}, s^{\prime}\right)\right]=0,} \\
{\left[\widehat{B}^{-}\left(x_{1}, x_{2}\right), \widehat{B}^{+}\left(x_{1}^{\prime}, x_{2}^{\prime}\right)\right]=\delta\left(x_{1}-x_{1}^{\prime}\right) \delta\left(x_{2}-x_{2}^{\prime}\right),} \\
{\left[\widehat{B}^{ \pm}\left(x_{1}, x_{2}\right), \widehat{B}^{ \pm}\left(x_{1}^{\prime}, x_{2}^{\prime}\right)\right]=0,} \\
{\left[\hat{b}^{ \pm}(x, s), \widehat{B}^{ \pm}\left(x_{1}^{\prime}, x_{2}^{\prime}\right)\right]=\left[\hat{b}^{ \pm}(x, s), \widehat{B}^{\mp}\left(x_{1}^{\prime}, x_{2}^{\prime}\right)\right]=0 .}
\end{gathered}
$$

Пусть $\Phi_{0}-$ вакуумный вектор в пространстве $\mathcal{F}$, обладающий следующими свойствами:

$$
\hat{b}^{-}(x, s) \Phi_{0}=0, \quad \widehat{B}^{-}\left(x_{1}, x_{2}\right) \Phi_{0}=0 .
$$

Переменная $x$ лежит на трехмерном торе $L \times L \times L$, который будем обозначать $\mathbf{T}$. Дискретная переменная $s=0,1, \ldots$ называется номером или статистическим спином. Любой вектор $\Phi$ пространства $\mathcal{F}$ единственным образом представляется в виде

$$
\begin{aligned}
\Phi=\sum_{k=0}^{\infty} & \sum_{M=0}^{\infty} \frac{1}{k ! M !} \sum_{s_{1}=0}^{\infty} \ldots \sum_{s_{k}=0}^{\infty} \int \ldots \int d x_{1} \ldots d x_{k} d y_{1} \ldots d y_{2 M} \times \\
& \times \Phi_{k, M}\left(x_{1}, s_{1} ; \ldots ; x_{k}, s_{k} ; y_{1}, y_{2} ; \ldots ; y_{2 M-1}, y_{2 M}\right) \times \\
& \times \hat{b}^{+}\left(x_{1}, s_{1}\right) \ldots \hat{b}^{+}\left(x_{k}, s_{k}\right) \widehat{B}^{+}\left(y_{1}, y_{2}\right) \ldots \widehat{B}^{+}\left(y_{2 M-1}, y_{2 M}\right) \Phi_{0}
\end{aligned}
$$


где функция $\Phi_{k, M}\left(x_{1}, s_{1} ; \ldots ; x_{k}, s_{k} ; y_{1}, y_{2} ; \ldots ; y_{2 M-1}, y_{2 M}\right)$ симметрична относительно перестановок пар переменных $\left(x_{j}, s_{j}\right)$ и $\left(x_{i}, s_{i}\right)$ и относительно перестановок пар переменных $\left(y_{2 j-1}, y_{2 j}\right)$ и $\left(y_{2 i-1}, y_{2 i}\right)$. В бозонном случае вводится подпространство $\mathcal{F}_{k, M}^{\text {Symm }}$, состоящее из векторов $\Phi$, у которых $\Phi_{k^{\prime}, M^{\prime}}=0$ при $\left(k^{\prime}, M^{\prime}\right) \neq(k, M)$, а $\Phi_{k, M}$ является симметричной функцией переменных $x_{1}, x_{2}, \ldots, x_{k}, y_{1}, y_{2}, \ldots, y_{2 M}$. В фермионном случае вводится подпространство $\mathcal{F}_{k, M}^{\text {Asymm }}$, состоящее из векторов $\Phi$ таких, что $\Phi_{k^{\prime}, M^{\prime}}=0$ при $\left(k^{\prime}, M^{\prime}\right) \neq(k, M)$ и $\Phi_{k, M}$ - антисимметричная функция переменных $x_{1}, x_{2}, \ldots, x_{k}, y_{1}, y_{2}, \ldots, y_{2}$. Ортогональный проектор пространства $\mathcal{F}$ на подпространство $\mathcal{F}_{k, M}^{\mathrm{Symm}}$ имеет вид [6]-[11], [13]-[18]

$$
\begin{aligned}
\widehat{\Pi}_{k, M}^{\text {Symm }} & =\frac{1}{k ! M !} \sum_{s_{1}=0}^{\infty} \ldots \sum_{s_{k}=0}^{\infty} \int \ldots \int d x_{1} \ldots d x_{k} d y_{1} \ldots d y_{2 M} \times \\
& \times \hat{b}^{+}\left(x_{1}, s_{1}\right) \ldots \hat{b}^{+}\left(x_{k}, s_{k}\right) \widehat{B}^{+}\left(y_{1}, y_{2}\right) \ldots \widehat{B}^{+}\left(y_{2 M-1}, y_{2 M}\right) \times \\
& \times \underset{x_{1} \ldots x_{k} y_{1} \ldots y_{2 M}}{\operatorname{Symm}}\left(\hat{b}^{-}\left(x_{1}, s_{1}\right) \ldots \hat{b}^{-}\left(x_{k}, s_{k}\right) \widehat{B}^{-}\left(y_{1}, y_{2}\right) \ldots \widehat{B}^{-}\left(y_{2 M-1}, y_{2 M}\right)\right) \times \\
& \times \exp \left(-\sum_{s=0}^{\infty} \int d x \hat{b}^{+}(x, s) \hat{b}^{-}(x, s)-\iint d y d y^{\prime} \widehat{B}^{+}\left(y, y^{\prime}\right) \widehat{B}^{-}\left(y, y^{\prime}\right)\right),
\end{aligned}
$$

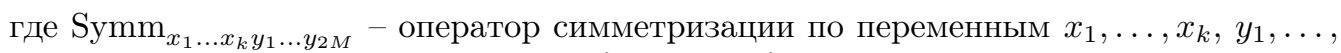
$y_{2 M}$, а операторы $\hat{b}^{+}(x, s), \hat{b}^{-}(x, s), \widehat{B}^{+}\left(y, y^{\prime}\right), \widehat{B}^{-}\left(y, y^{\prime}\right)$ упорядочены по Вику [19]. Ортогональный проектор пространства $\mathcal{F}$ на подпространство $\mathcal{F}_{k, M}^{\text {Asymm }}$ имеет вид [6]

$$
\begin{aligned}
& \widehat{\Pi}_{k, M}^{\text {Asymm }}=\frac{1}{k ! M !} \sum_{s_{1}=0}^{\infty} \ldots \sum_{s_{k}=0}^{\infty} \int \ldots \int d x_{1} \ldots d x_{k} d y_{1} \ldots d y_{2 M} \times \\
& \quad \times \hat{b}^{+}\left(x_{1}, s_{1}\right) \ldots \hat{b}^{+}\left(x_{k}, s_{k}\right) \widehat{B}^{+}\left(y_{1}, y_{2}\right) \ldots \widehat{B}^{+}\left(y_{2 M-1}, y_{2 M}\right) \times \\
& \quad \times \underset{x_{1} \ldots x_{k} y_{1} \ldots y_{2 M}}{\operatorname{Asymm}}\left(\hat{b}^{-}\left(x_{1}, s_{1}\right) \ldots \hat{b}^{-}\left(x_{k}, s_{k}\right) \widehat{B}^{-}\left(y_{1}, y_{2}\right) \ldots \widehat{B}^{-}\left(y_{2 M-1}, y_{2 M}\right)\right) \times \\
& \quad \times \exp \left(-\sum_{s=0}^{\infty} \int d x \hat{b}^{+}(x, s) \hat{b}^{-}(x, s)-\iint d y d y^{\prime} \widehat{B}^{+}\left(y, y^{\prime}\right) \widehat{B}^{-}\left(y, y^{\prime}\right)\right),
\end{aligned}
$$

где Asymm $_{x_{1} \ldots x_{k} y_{1} \ldots y_{2} \text { m }}$ - оператор антисимметризации по переменным $x_{1}, \ldots, x_{k}, y_{1}$, $\ldots, y_{2 M}$. Здесь и везде далее, если не оговорено особо, операторы $\hat{b}^{+}(x, s), \hat{b}^{-}(x, s)$, $\widehat{B}^{+}\left(y, y^{\prime}\right), \widehat{B}^{-}\left(y, y^{\prime}\right)$ упорядочены по Вику.

Рассмотрим систему $N$ тождественных частиц на торе $\mathbf{T}$. Будем считать, что гамильтониан $N$ бозонов или фермионов имеет вид

$$
\widehat{H}_{N}=-\frac{\hbar^{2}}{2 m} \sum_{j=1}^{N} \Delta_{j}+\sum_{j=1}^{N} \sum_{l=j+1}^{N} V\left(x_{j}-x_{l}\right) .
$$

Согласно [6] этому оператору в бозонном случае отвечает ультравторично-квантованный гамильтониан следующего вида:

$$
\overline{\widehat{H}}_{\mathrm{B}}=\sum_{k=0}^{\infty} \sum_{M=0}^{\infty} \frac{1}{k ! M !} \sum_{s_{1}=0}^{\infty} \ldots \sum_{s_{k}=0}^{\infty} \int \ldots \int d x_{1} \ldots d x_{k} d y_{1} \ldots d y_{2 M} \times
$$




$$
\begin{aligned}
& \times \hat{b}^{+}\left(x_{1}, s_{1}\right) \ldots \hat{b}^{+}\left(x_{k}, s_{k}\right) \widehat{B}^{+}\left(y_{1}, y_{2}\right) \ldots \widehat{B}^{+}\left(y_{2 M-1}, y_{2 M}\right) \widehat{H}_{k+2 M} \times \\
& \times \underset{x_{1} \ldots x_{k} y_{1} \ldots y_{2 M}}{\operatorname{Symm}}\left(\hat{b}^{-}\left(x_{1}, s_{1}\right) \ldots \hat{b}^{-}\left(x_{k}, s_{k}\right) \widehat{B}^{-}\left(y_{1}, y_{2}\right) \ldots \widehat{B}^{-}\left(y_{2 M-1}, y_{2 M}\right)\right) \times \\
& \times \exp \left(-\sum_{s=0}^{\infty} \int d x \hat{b}^{+}(x, s) \hat{b}^{-}(x, s)-\iint d y d y^{\prime} \widehat{B}^{+}\left(y, y^{\prime}\right) \widehat{B}^{-}\left(y, y^{\prime}\right)\right),
\end{aligned}
$$

а в фермионном случае соответствующий оператор $\widehat{\widehat{H}}_{\mathrm{F}}$ выражается аналогичной формулой, в которой оператор Symm заменяется на Asymm. По аналогии с (13) и (14) ультравторично-квантованный оператор $\bar{A}$ сопоставляется [6] любому $N$-частичному оператору

$$
\widehat{A}_{N}\left(x_{1}^{2}, \ldots, x_{N}^{2} ;-i \frac{1}{\partial x_{1}}, \ldots,-i \frac{\frac{1}{\partial}}{\partial x_{N}}\right) .
$$

Например, единичному оператору в бозонном случае сопоставляется ультравторично-квантованный единичный оператор вида

$$
\begin{aligned}
\widehat{\vec{E}}_{\mathrm{B}}=\sum_{k=0}^{\infty} & \sum_{M=0}^{\infty} \frac{1}{k ! M !} \sum_{s_{1}=0}^{\infty} \ldots \sum_{s_{k}=0}^{\infty} \int \ldots \int d x_{1} \ldots d x_{k} d y_{1} \ldots d y_{2 M} \times \\
& \times \hat{b}^{+}\left(x_{1}, s_{1}\right) \ldots \hat{b}^{+}\left(x_{k}, s_{k}\right) \widehat{B}^{+}\left(y_{1}, y_{2}\right) \ldots \widehat{B}^{+}\left(y_{2 M-1}, y_{2 M}\right) \times \\
& \times \underset{x_{1} \ldots x_{k} y_{1} \ldots y_{2 M}}{\operatorname{Symm}}\left(\hat{b}^{-}\left(x_{1}, s_{1}\right) \ldots \hat{b}^{-}\left(x_{k}, s_{k}\right) \widehat{B}^{-}\left(y_{1}, y_{2}\right) \ldots \widehat{B}^{-}\left(y_{2 M-1}, y_{2 M}\right)\right) \times \\
& \times \exp \left(-\sum_{s=0}^{\infty} \int d x \hat{b}^{+}(x, s) \hat{b}^{-}(x, s)-\iint d y d y^{\prime} \widehat{B}^{+}\left(y, y^{\prime}\right) \widehat{B}^{-}\left(y, y^{\prime}\right)\right)
\end{aligned}
$$

который есть сумма проекторов (12). Аналогично ультравторично-квантованный единичный оператор в фермионном случае есть

$$
\widehat{\widehat{E}}_{\mathrm{F}}=\sum_{k=0}^{\infty} \sum_{M=0}^{\infty} \widehat{\Pi}_{k, M}^{\mathrm{Asymm}},
$$

а в формуле (15) оператор Symm заменяется на Asymm.

Рассмотрим следующую задачу на собственные значения:

$$
\widehat{\widehat{H}}_{\mathrm{B}, \mathrm{F}} \Phi=\lambda \widehat{\widehat{E}}_{\mathrm{B}} \Phi, \quad \overline{\widehat{E}} \Phi \neq 0,
$$

в бозонном и фермионном случаях. Справедливо утверждение, доказанное в [6]: на подпространствах $\mathcal{F}_{k, M}^{\mathrm{Symm}}$ u $\mathcal{F}_{k, M}^{\mathrm{Asymm}}$ пространства $\mathcal{F}$ операторы $\widehat{\widehat{H}}_{\mathrm{B}} u \overline{\widehat{H}}_{\mathrm{F}}$, соответственно, совпадают с оператором $\widehat{H}_{k+2 M}$, если подействовать на них проектором на $N$-мерное подпространство (см. бормулу (22) ниже). Поэтому собственные значения $\lambda$ задачи (16) в бозонном и фермионном случаях совпадают с соответствующими собственными значениями операторов $\widehat{H}_{N}(13)$. В случае, когда коммутаторы между операторами $\hat{b}^{-}(x, s)$ и $\hat{b}^{+}(x, s)$, а также $\widehat{B}^{-}(x, y)$ и $\widehat{B}^{+}(x, y)$ 
имеют порядок малости $1 / N$, асимптотика решений задачи (16) определяется точками экстремума псевдосимвола, отвечающего задаче (16). Для произвольного вторично-квантованного оператора $\widehat{A}$ псевдосимвол отвечающего ему ультравторично-квантованного оператора $\widehat{A}$ выражается следующей формулой [6]:

$$
A_{\mathrm{B}, \mathrm{F}}\left[b^{*}(\cdot), b(\cdot), B^{*}(\cdot), B(\cdot)\right]=\frac{\operatorname{Sp}\left(\widehat{\rho}_{\mathrm{B}, \mathrm{F}} \widehat{A}\right)}{\operatorname{Sp}\left(\widehat{\rho}_{\mathrm{B}, \mathrm{F}}\right)} .
$$

В бозонном случае псевдосимвол имеет вид

$$
\begin{aligned}
\mathcal{H}_{\mathrm{B}}\left[b^{*}(\cdot), b(\cdot), B^{*}(\cdot), B(\cdot)\right]= \\
\quad=\left\{\sum_{k, M=0}^{\infty} \frac{1}{k ! M !} \sum_{s_{1}=0}^{\infty} \ldots \sum_{s_{k}=0}^{\infty} \int \ldots \int d x_{1} \ldots d x_{k} d y_{1} \ldots d y_{2 M} \times\right. \\
\quad \times b^{*}\left(x_{1}, s_{1}\right) \ldots b^{*}\left(x_{k}, s_{k}\right) B^{*}\left(y_{1}, y_{2}\right) \ldots B^{*}\left(y_{2 M-1}, y_{2 M}\right) H_{k+2 M} \times \\
\left.\quad \times \underset{x_{1} \ldots x_{k} y_{1} \ldots y_{2 M}}{\operatorname{Symm}}\left(b\left(x_{1}, s_{1}\right) \ldots b\left(x_{k}, s_{k}\right) B\left(y_{1}, y_{2}\right) \ldots B\left(y_{2 M-1}, y_{2 M}\right)\right)\right\} \times \\
\quad \times\left\{\sum_{k^{\prime}, M^{\prime}=0}^{\infty} \frac{1}{k^{\prime} ! M^{\prime} !} \sum_{s_{1}^{\prime}=0}^{\infty} \ldots \sum_{s_{k^{\prime}}^{\prime}=0}^{\infty} \int \ldots \int d x_{1}^{\prime} \ldots d x_{k^{\prime}}^{\prime} d y_{1}^{\prime} \ldots d y_{2 M^{\prime}}^{\prime} \times\right. \\
\quad \times b^{*}\left(x_{1}^{\prime}, s_{1}^{\prime}\right) \ldots b^{*}\left(x_{k^{\prime}}^{\prime}, s_{k^{\prime}}^{\prime}\right) B^{*}\left(y_{1}^{\prime}, y_{2}^{\prime}\right) \ldots B^{*}\left(y_{2 M^{\prime}-1}^{\prime}, y_{2 M^{\prime}}^{\prime}\right) \times \\
\left.\quad \times \underset{x_{1}^{\prime} \ldots x_{k^{\prime}}^{\prime} y_{1}^{\prime} \ldots y_{2 M^{\prime}}^{\prime}}{\operatorname{Symm}}\left(b\left(x_{1}^{\prime}, s_{1}^{\prime}\right) \ldots b\left(x_{k^{\prime}}^{\prime}, s_{k^{\prime}}^{\prime}\right) B\left(y_{1}^{\prime}, y_{2}^{\prime}\right) \ldots B\left(y_{2 M^{\prime}-1}^{\prime}, y_{2 M^{\prime}}^{\prime}\right)\right)\right\}^{-1} .
\end{aligned}
$$

В фермионном случае псевдосимвол выражается аналогично, только Symm в формуле (17) заменяется на Asymm. Следующее тождество для псевдосимвола (17) имеет место в бозонном случае:

$$
\mathcal{H}_{\mathrm{B}}\left[b^{*}(\cdot), b(\cdot), B^{*}(\cdot), B(\cdot)\right]=\frac{\operatorname{Sp}\left(\widehat{\rho}_{\mathrm{B}} \widehat{H}\right)}{\operatorname{Sp}\left(\widehat{\rho}_{\mathrm{B}}\right)},
$$

где $\widehat{H}, \widehat{\rho}_{\mathrm{B}}-$ вторично-квантованные операторы:

$$
\begin{aligned}
\widehat{H}=\int & d x \widehat{\psi}^{+}(x)\left(-\frac{\hbar^{2}}{2 m} \Delta\right) \widehat{\psi}^{-}(x)+ \\
& +\frac{1}{2} \iint d x d y V(x, y) \widehat{\psi}^{+}(y) \widehat{\psi}^{+}(x) \widehat{\psi}^{-}(y) \widehat{\psi}^{-}(x) .
\end{aligned}
$$

Здесь $\widehat{\rho}_{\mathrm{B}}$ зависит от функций $b(x, s), B\left(y, y^{\prime}\right)$ :

$$
\begin{aligned}
\widehat{\rho}_{\mathrm{B}}=\sum_{k=0}^{\infty} & \sum_{M=0}^{\infty} \frac{1}{k ! M !(k+2 M) !}\left(\sum_{s=0}^{\infty} \iint d x d x^{\prime} b(x, s) b^{*}\left(x^{\prime}, s\right) \widehat{\psi}^{+}(x) \widehat{\psi}^{-}\left(x^{\prime}\right)\right)^{k} \times \\
& \times\left(\iint d y_{1} d y_{2} B\left(y_{1}, y_{2}\right) \widehat{\psi}^{+}\left(y_{1}\right) \widehat{\psi}^{+}\left(y_{2}\right)\right)^{M} \times \\
& \times\left(\iint d y_{1}^{\prime} d y_{2}^{\prime} B\left(y_{1}^{\prime}, y_{2}^{\prime}\right) \widehat{\psi}^{-}\left(y_{1}^{\prime}\right) \widehat{\psi}^{-}\left(y_{2}^{\prime}\right)\right)^{M} \exp \left(-\int d z \widehat{\psi}^{+}(z) \widehat{\psi}^{-}(z)\right)
\end{aligned}
$$


где $\widehat{\psi}^{+}(x), \widehat{\psi}^{-}(x)$ - бозонные операторы рождения и уничтожения, упорядоченные по Вику [19]. В фермионном случае имеет место аналогичное тождество

$$
\mathcal{H}_{\mathrm{F}}\left[b^{*}(\cdot), b(\cdot), B^{*}(\cdot), B(\cdot)\right]=\frac{\operatorname{Sp}\left(\widehat{\rho}_{\mathrm{F}} \widehat{H}\right)}{\operatorname{Sp}\left(\widehat{\rho}_{\mathrm{F}}\right)}
$$

где $\widehat{H}, \widehat{\rho}_{\mathrm{F}}$ - следующие вторично-квантованные операторы:

$$
\begin{aligned}
\widehat{H}= & \int d x \widehat{\psi}^{+}(x)\left(-\frac{\hbar^{2}}{2 m} \Delta\right) \widehat{\psi}^{-}(x)+\frac{1}{2} \iint d x d y V(x, y) \widehat{\psi}^{+}(x) \widehat{\psi}^{+}(y) \widehat{\psi}^{-}(y) \widehat{\psi}^{-}(x), \\
\widehat{\rho}_{\mathrm{F}}= & \sum_{k=0}^{\infty} \sum_{M=0}^{\infty} \frac{1}{k ! M !(k+2 M) !}\left(\iint d y_{1} d y_{2} B\left(y_{1}, y_{2}\right) \widehat{\psi}^{+}\left(y_{1}\right) \widehat{\psi}^{+}\left(y_{2}\right)\right)^{M} \times \\
& \times \sum_{s_{1}=0}^{\infty} \cdots \sum_{s_{k}=0}^{\infty} \int \ldots \int d x_{1} d x_{1}^{\prime} \ldots d x_{k} d x_{k}^{\prime} b\left(x_{1}, s_{1}\right) b^{*}\left(x_{1}^{\prime}, s_{1}\right) \ldots b\left(x_{k}, s_{k}\right) b^{*}\left(x_{k}^{\prime}, s_{k}\right) \times \\
& \times \widehat{\psi}^{+}\left(x_{1}\right) \ldots \widehat{\psi}^{+}\left(x_{k}\right) \widehat{P}_{0} \widehat{\psi}^{-}\left(x_{k}^{\prime}\right) \ldots \widehat{\psi}^{-}\left(x_{1}^{\prime}\right)\left(\iint d y_{1}^{\prime} d y_{2}^{\prime} B\left(y_{1}^{\prime}, y_{2}^{\prime}\right) \widehat{\psi}^{-}\left(y_{2}^{\prime}\right) \widehat{\psi}^{-}\left(y_{1}^{\prime}\right)\right)^{M},
\end{aligned}
$$

где в данном случае $\widehat{\psi}^{+}(x), \widehat{\psi}^{-}(x)$ - фермионные операторы рождения и уничтожения, $\widehat{P}_{0}$ - проектор на вакуумный вектор фермионного фоковского пространства.

В пространстве $\mathcal{F}$ вводятся [6] ультравторично-квантованные операторы числа частиц

$$
\widehat{\widehat{N}}_{\mathrm{B}}=\sum_{k=0}^{\infty} \sum_{M=0}^{\infty}(k+2 M) \widehat{\Pi}_{k, M}^{\mathrm{Symm}}, \quad \widehat{\widehat{N}}_{\mathrm{F}}=\sum_{k=0}^{\infty} \sum_{M=0}^{\infty}(k+2 M) \widehat{\Pi}_{k, M}^{\mathrm{Asymm}} .
$$

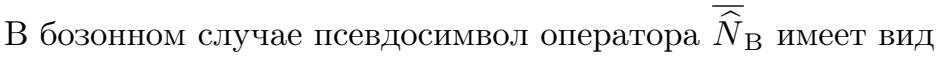

$$
\begin{aligned}
N_{\mathrm{B}} & =\left\{\sum_{k=0}^{\infty} \sum_{M=0}^{\infty} \frac{k+2 M}{k ! M !} \sum_{s_{1}=0}^{\infty} \ldots \sum_{s_{k}=0}^{\infty} \int \ldots \int d x_{1} \ldots d x_{k+2 M} \times\right. \\
& \times b^{*}\left(x_{1}, s_{1}\right) \ldots b^{*}\left(x_{k}, s_{k}\right) B^{*}\left(x_{k+1}, x_{k+2}\right) \ldots B^{*}\left(x_{k+2 M-1}, x_{k+2 M}\right) \times \\
& \left.\times \underset{x_{1} \ldots x_{k+2 M}}{\operatorname{Symm}}\left(b\left(x_{1}, s_{1}\right) \ldots b\left(x_{k}, s_{k}\right) B\left(x_{k+1}, x_{k+2}\right) \ldots B\left(x_{k+2 M-1}, x_{k+2 M}\right)\right)\right\} \times \\
& \times\left\{\sum_{k^{\prime}=0}^{\infty} \sum_{M^{\prime}=0}^{\infty} \frac{1}{k^{\prime} ! M^{\prime} !} \sum_{s_{1}^{\prime}=0}^{\infty} \ldots \sum_{s_{k^{\prime}}^{\prime}=0}^{\infty} \int \ldots \int d z_{1} \ldots d z_{k^{\prime}+2 M^{\prime}} b^{*}\left(z_{1}, s_{1}^{\prime}\right) \ldots b^{*}\left(z_{k^{\prime}}, s_{k^{\prime}}^{\prime}\right) \times\right. \\
& \times B^{*}\left(z_{k^{\prime}+1}, z_{k^{\prime}+2}\right) \ldots B^{*}\left(x_{k^{\prime}+2 M^{\prime}-1}, x_{k^{\prime}+2 M^{\prime}}\right) \times \\
& \left.\times \underset{z_{1} \ldots z_{k^{\prime}+2 M^{\prime}}}{\operatorname{Symm}}\left(b\left(z_{1}, s_{1}^{\prime}\right) \ldots B\left(z_{k^{\prime}+2 M^{\prime}-1}, z_{k^{\prime}+2 M^{\prime}}\right)\right)\right\}^{-1} .
\end{aligned}
$$

В соответствующей формуле в фермионном случае Symm заменяется на Asymm. 


\section{3. СИМВОЛ УЛЬТРАВТОРИЧНО-КВАНТОВАННОГО ОПЕРАТОРА}

Заметим прежде всего, что определение псевдосимвола, данное выше, не совсем отражает термодинамическую асимптотику, хотя это определение и согласуется с правилом Боголюбова-Дирака, согласно которому операторы рождения и уничтожения в главном асимптотическом члене надо положить $c$-числами. Поэтому определим символ правильно (введем истинный символ). Пусть оператор $\widehat{H}$ имеет вид

$$
\begin{aligned}
\widehat{H}=\sum_{l=1}^{L} \int \ldots \int d x_{1} \ldots d x_{l} \widehat{\psi}^{+}\left(x_{1}\right) \ldots \widehat{\psi}^{+}\left(x_{l}\right) \times \\
\quad \times H_{l}\left(x_{1}^{2}, \ldots, x_{l}^{2} ;-i \frac{\frac{1}{\partial}}{\partial x_{1}}, \ldots,-i \frac{\partial}{\partial x_{l}}\right) \widehat{\psi}^{-}\left(x_{l}\right) \ldots \widehat{\psi}^{-}\left(x_{1}\right) .
\end{aligned}
$$

Тогда в случае ультравторичного квантования без операторов рождения и уничтожения пар частиц $\widehat{B}^{ \pm}(x, y)$ для операторов $\widehat{\widehat{H}}$ и $\widehat{E}$, определенных выше, имеет место тождество

$$
\widehat{\widehat{H}}=\widehat{\widehat{E} \widehat{A}}
$$

где $\overline{\widehat{A}}$ - некоторый оператор в пространстве $\mathcal{F}$, который задается равенством

$$
\begin{gathered}
\overline{\widehat{A}}=\sum_{l=1}^{L} \sum_{s_{1}=0}^{\infty} \ldots \sum_{s_{l}=0}^{\infty} \int \ldots \int d x_{1} \ldots d x_{l} \hat{b}^{+}\left(x_{1}, s_{1}\right) \ldots \widehat{\psi}^{+}\left(x_{l}, s_{l}\right) \times \\
\quad \times H_{l}\left(x_{1}^{2}, \ldots, x_{l} ;-i \frac{\partial}{\partial x_{1}}, \ldots,-i \frac{\partial}{\partial x_{l}}\right) \hat{b}^{-}\left(x_{l}, s_{l}\right) \ldots \hat{b}^{-}\left(x_{1}\right) .
\end{gathered}
$$

В случае, если ультравторичное квантование учитывает также операторы рождения и уничтожения пар частиц, тождество (25) справедливо, но оператор $\widehat{\widehat{A}}$ имеет более сложный вид, чем (26). Например, если оператор $\widehat{H}$ является частным случаем оператора $(24)$ при $L=2$, оператор $\widehat{\widehat{A}}$ имеет вид

$$
\begin{aligned}
\widehat{A}=\sum_{s=0}^{\infty} & \int d x \hat{b}^{+}(x, s)\left(-\frac{h^{2}}{2 m} \Delta\right) \hat{b}^{-}(x, s)+ \\
& +\iint d x d y \widehat{B}^{+}(x, y)\left(-\frac{h^{2}}{2 m}\left(\Delta_{x}+\Delta_{y}\right)\right) \widehat{B}^{-}(x, y)+ \\
& +\frac{1}{2} \sum_{s_{1}=0}^{\infty} \sum_{s_{2}=0}^{\infty} \iint d x d y V(x, y) \hat{b}^{+}\left(x, s_{1}\right) \hat{b}^{+}\left(y, s_{2}\right) \hat{b}^{-}\left(y, s_{2}\right) \hat{b}^{-}\left(x, s_{1}\right)+ \\
& +\sum_{s=0}^{\infty} \iiint d x d y d z(V(x, y)+V(x, z)) \hat{b}^{+}(x, s) \widehat{B}^{+}(y, z) \widehat{B}^{-}(y, z) \hat{b}^{-}(x, s)+ \\
& +\iint d x d y V(x, y) \widehat{B}^{+}(x, y) \widehat{B}^{-}(x, y)+
\end{aligned}
$$




$$
\begin{aligned}
& +\frac{1}{2} \iiint \int d x d y d z d w V(x, y) \widehat{B}^{+}(x, y) \widehat{B}^{+}(z, w) \times \\
& \times\left(\widehat{B}^{-}(y, w) \widehat{B}^{-}(x, z)+\widehat{B}^{-}(w, y) \widehat{B}^{-}(z, x)+\right. \\
& \left.+\widehat{B}^{-}(y, z) \widehat{B}^{-}(w, x)+\widehat{B}^{-}(z, y) \widehat{B}^{-}(x, w)\right) .
\end{aligned}
$$

Если в выражениях (26), (27) положить операторы $\widehat{B}^{ \pm}(x, y), \hat{b}^{ \pm}(x, y) c$-числами, то получится (истинный) символ, который отвечает асимптотике в термодинамическом пределе.

\section{4. СЛУЧАЙ ЧЕТНОГО ЧИСЛА НЕЙТРОНОВ}

Рассмотрим систему $N$ тождественных бозонов массы $m$, которые находятся на торе $\mathbf{T}$, диаметры которого равны $L_{1}, L_{2}, L_{2}$. Будем считать, что бозоны взаимодействуют между собой и потенциал взаимодействия имеет вид

$$
V\left(N^{1 / 3}(x-y)\right)
$$

где $V(\xi)$ - финитная четная функция, $x, y$ - координаты бозонов на торе Т. Граничные условия вдоль стороны $L_{1}$ будем считать периодическими, а вдоль $L_{2}$ наложим условия равенства производных нулю. Отметим, что потенциал взаимодействия (28) зависит от $N$ так, что его радиус уменьшается с увеличением числа частиц $N$, при этом в среднем число частиц, с которыми взаимодействует одна частица, остается постоянным.

При ультравторичном квантовании по парам рассматриваемой бозонной системе соответствует ультравторично-квантованный оператор $\overline{\widehat{H}}$, явный вид которого приведен в (24). Как обсуждалось выше, для этого ультравторично-квантованного оператора имеет место тождество

$$
\widehat{\widehat{H}}=\widehat{\widehat{E}} \widehat{A},
$$

где $\widehat{\widehat{E}}$ - ультравторично-квантованный единичный оператор, а $\widehat{A}$ - оператор в пространстве ультравторичного квантования. Нетрудно убедиться, что тождеству (29) удовлетворяет оператор следующего вида:

$$
\begin{aligned}
\widehat{A} & =\iint d x d y \widehat{B}^{+}(x, y)\left(-\frac{\hbar^{2}}{2 m}\left(\Delta_{x}+\Delta_{y}\right)+V\left(N^{1 / 3}(x-y)\right)\right) \widehat{B}^{-}(x, y)+ \\
& +2 \iiint \int d x d y d x^{\prime} d y^{\prime} V\left(N^{1 / 3}(x-y)\right) \widehat{B}^{+}(x, y) \widehat{B}^{+}\left(x^{\prime}, y^{\prime}\right) \widehat{B}^{-}\left(x, x^{\prime}\right) \widehat{B}^{-}\left(y, y^{\prime}\right),
\end{aligned}
$$

где $\widehat{B}^{+}(x, y)$ и $\widehat{B}^{-}(x, y)$ - соответственно бозонные операторы рождения и уничтожения пары частиц в фоковском пространстве ультравторичного квантования. В силу тождества (29) для нахождения асимптотики спектра рассматриваемой бозонной системы в пределе при $N \rightarrow \infty$ нужно найти соответствующую асимптотику для оператора (30).

Так как функция (28), умноженная на $N$, в пределе при $N \rightarrow \infty$ в слабом смысле сходится к дельта-функции Дирака, то в операторе (30) при втором слагаемом в этом 
предельном случае стоит малый параметр $1 / N$. Это означает, что для нахождения асимптотики собственных значений и собственных функций оператора $\widehat{A}$ можно применить квазиклассические методы, развитые в [4]. Асимптотика собственных значений и собственных функций определяется символом оператора (30), этот символ является истинным символом ультравторично-квантованной задачи. Истинным символом, соответствующим оператору (30), является следующий функционал, определенный для пары функций $\Phi^{+}(x, y)$ и $\Phi(x, y)$ :

$$
\begin{aligned}
& \mathcal{H}\left[\Phi^{+}(\cdot), \Phi(\cdot)\right]=\iint d x d y \Phi^{+}(x, y)\left(-\frac{\hbar^{2}}{2 m}\left(\Delta_{x}+\Delta_{y}\right)\right) \Phi(x, y)+ \\
& \quad+2 \iiint \int d x d y d x^{\prime} d y^{\prime}\left(N V\left(N^{1 / 3}(x-y)\right)\right) \Phi^{+}(x, y) \Phi^{+}\left(x^{\prime}, y^{\prime}\right) \Phi\left(x, x^{\prime}\right) \Phi\left(y, y^{\prime}\right) .
\end{aligned}
$$

Из закона сохранения числа частиц в системе следует условие для функций $\Phi^{+}(x, y)$ и $\Phi(x, y)$

$$
\iint d x d y \Phi^{+}(x, y) \Phi(x, y)=\frac{1}{2} .
$$

Согласно асимптотическим методам [4] каждому решению системы уравнений

$$
\Omega \Phi(x, y)=\frac{\delta \mathcal{H}}{\delta \Phi^{+}(x, y)}, \quad \Omega \Phi^{+}(x, y)=\frac{\delta \mathcal{H}}{\delta \Phi(x, y)}
$$

удовлетворяющему также условию (32), в пределе при $N \rightarrow \infty$ соответствует асимптотическая серия собственных функций и собственных значений оператора (30). Из явного вида истинного символа (31) следует, что система уравнений (33) записывается как

$$
\begin{aligned}
& \Omega \Phi(x, y)=-\frac{\hbar^{2}}{2 m}\left(\Delta_{x}+\Delta_{y}\right) \Phi(x, y)+ \\
& \quad+\iint d x^{\prime} d y^{\prime}\left(N V\left(N^{1 / 3}(x-y)\right)+N V\left(N^{1 / 3}\left(x^{\prime}-y^{\prime}\right)\right)\right) \Phi^{+}\left(x^{\prime}, y^{\prime}\right) \Phi\left(x, x^{\prime}\right) \Phi\left(y, y^{\prime}\right), \\
& \Omega \Phi^{+}(x, y)=-\frac{\hbar^{2}}{2 m}\left(\Delta_{x}+\Delta_{y}\right) \Phi^{+}(x, y)+ \\
& \quad+2 \iint d x^{\prime} d y^{\prime}\left(N V\left(N^{1 / 3}\left(x-x^{\prime}\right)\right)+N V\left(N^{1 / 3}\left(y-y^{\prime}\right)\right)\right) \Phi\left(x^{\prime}, y^{\prime}\right) \Phi^{+}\left(x, x^{\prime}\right) \Phi^{+}\left(y, y^{\prime}\right) .
\end{aligned}
$$

Пусть $v_{q}$ - коэффициенты разложения в ряд Фурье на торе $\left(L_{1}, L_{2}, L_{2}\right)$ потенциала $N V\left(N^{1 / 3} x\right)$ :

$$
v_{q}=\frac{1}{L_{1} L_{2}^{2}} \int_{\mathbf{T}} e^{-i q x} N V(\sqrt[3]{N} x) d x, \quad v_{-q}=v_{q} .
$$

Точным решением системы (34) будут следующие функции:

$$
\begin{aligned}
\Phi_{k_{1}, k_{2}}^{+} & =\frac{1}{L_{1} L_{2}^{2}} e^{-i k_{1}(x+y)} \cos \left(k_{2}(x-y)\right), \\
\Phi_{k_{1}, k_{2}} & =\frac{1}{L_{1} L_{2}^{2}} \sum_{l=0}^{\infty} \varphi_{k_{2}, l} e^{i k_{1}(x+y)} e^{i l(x-y)}
\end{aligned}
$$


с собственным значением

$$
\Omega=\frac{h^{2}}{m}\left(k_{1}^{2}+k_{2}^{2}\right)+v_{0}+v_{2 k_{2}}
$$

где функция $\varphi_{k_{2}, l}$ имеет вид

$$
\varphi_{k_{2}, l}= \begin{cases}-\frac{b_{l}}{2}+\frac{1}{2} \sqrt{b_{l}^{2}-1}, & l^{2}>k_{2}^{2}, \\ -\frac{b_{l}}{2}-\frac{1}{2} \sqrt{b_{l}^{2}-1}, & l^{2}<k_{2}^{2}, \\ \frac{1}{2}, & l=k_{2},\end{cases}
$$

где

$$
b_{l}=\frac{\left(h^{2} / m\right)\left(l^{2}-k_{2}^{2}\right)-\left(v_{0}+v_{2 k_{2}}\right)}{v_{l-k_{2}}+v_{l+k_{2}}},
$$

причем $b_{l}=b_{-l}, \varphi_{k_{2}, l}=\varphi_{k_{2},-l}\left(\right.$ если $V(\xi) \rightarrow 0$, то $\left.\varphi_{k_{2}, l} \rightarrow 0\right)$. Векторы $k_{1}, k_{2}$ играют роль параметров, нумерующих различные решения этой системы; вектор $\hbar k_{1} / m$ равен скорости течения бозонной системы вдоль капилляра, вектор $k_{2}$ - волновой вектор поперечной моды.

Отметим, что $b_{l} \rightarrow \infty$ при $|l| \rightarrow \infty$, поскольку

$$
\left|v_{l}\right|=\frac{1}{L_{1} L_{2}^{2}} \int_{N \mathbf{T}} e^{-i l \xi / N} V(\xi) d \xi \leqslant \frac{1}{L_{1} L_{2}^{2}} \int_{N \mathbf{T}}|V(\xi)| d \xi<\frac{1}{L_{1} L_{2}^{2}} \int_{\mathbb{R}^{3}}|V(\xi)| d \xi
$$

(здесь $N \mathbf{T}$ - тор с диаметрами $N L_{1}, N L_{2}, N L_{2}$ ), а следовательно,

$$
\varphi_{k, l} \cong \frac{1}{b_{l}^{2}}
$$

и, значит, ряд (36) сходится абсолютно.

Разобьем ряд (36) на две части: суммы по $l \leqslant N^{1 / 6}$ и по $l>N^{1 / 6}$. Члены в первой сумме при $N \rightarrow \infty$ сходятся с точностью до $\left(N^{-1 / 6}\right)$ к

$$
\begin{aligned}
b_{l} & \rightarrow \frac{h^{2}\left(l^{2}-k_{2}^{2}\right)}{2 m V_{0}}-1 \stackrel{\text { def }}{=} b_{l}^{0}, \\
\varphi_{k_{2}, l} & \rightarrow-\frac{b_{l}}{2} \pm \frac{1}{2} \sqrt{b_{l}^{2}-1} \stackrel{\text { def }}{=} \varphi_{k_{2}, l}^{0} .
\end{aligned}
$$

Это легко получается после замены в (34) вида $\sqrt[3]{N} x=\xi$.

Вторая сумма стремится к нулю в силу (40) как $O\left(N^{-1 / 6}\right)$. Следовательно, в пределе при $N \rightarrow \infty$ система уравнений (34), дополненная условием (32), имеет при $k_{1}=0$ следующее семейство решений:

$$
\begin{aligned}
& \Phi_{k}^{+}(x, y)=\frac{1}{L_{1} L_{2}^{2}} \cos (k(x-y)), \\
& \Phi_{k}(x, y)=\frac{1}{L_{1} L_{2}^{2}} \sum_{l} \varphi_{k, l} e^{i l(x-y)},
\end{aligned}
$$


где $k$ и $l$ - трехмерные векторы вида $2 \pi\left(0, n_{2} / L_{2}, n_{3} / L_{2}\right), n_{2}, n_{3}$ - целые числа, $\varphi_{k, l}$ в формуле (38) в этом случае принимают вид

$$
\varphi_{k_{2}, l}^{0}=\frac{1}{2 V_{0}}\left(\frac{\hbar^{2}}{2 m}\left(k_{2}^{2}-l^{2}\right)+V_{0} \pm \sqrt{\left.\left(\frac{\hbar^{2}}{2 m}\left(k_{2}^{2}-l^{2}\right)+V_{0}\right)^{2}-V_{0}^{2}\right)},\right.
$$

где выбирается знак плюс в силу правила отбора; наконец, $V_{0}$ здесь обозначает величину

$$
V_{0}=\frac{1}{L_{1} L_{2}^{2}} \int_{\mathbb{R}^{3}} d x V(x) .
$$

Вектор $k$ в формулах (42) играет роль параметра, нумерующего различные решения системы уравнений (34), (32). Решения (42) представляют собой стоячие волны, и им соответствуют серии, в которых нет течения.

Главный член асимптотики собственных значений серии, соответствующей решению (35), (36), равен значению символа (31) на этих функциях, умноженному на $N$ :

$$
E_{k_{1}, k_{2}}=N\left(\frac{\hbar^{2}\left(k_{1}^{2}+k_{2}^{2}\right)}{2 m}+\frac{V_{0}}{2}\right) .
$$

Асимптотика собственных значений и собственных функций, в частности следующие после $E_{k_{1}, k_{2}}$ члены, помимо системы уравнений (34) определяется также решениями системы уравнений в вариациях для системы уравнений Гамильтона. Система уравнений в вариациях для (34) имеет вид

$$
\begin{aligned}
(\Omega-\lambda) F(x, y)=- & \frac{\hbar^{2}}{2 m}\left(\Delta_{x}+\Delta_{y}\right) F(x, y)+ \\
& +2 N \iint d x^{\prime} d y^{\prime}\left(V(\sqrt[3]{N}(x-y))+V\left(\sqrt[3]{N}\left(x^{\prime}-y^{\prime}\right)\right)\right) \times \\
& \times\left(G\left(x^{\prime}, y^{\prime}\right) \Phi\left(x, x^{\prime}\right) \Phi\left(y, y^{\prime}\right)+\Phi^{+}\left(x^{\prime}, y^{\prime}\right) F\left(x, x^{\prime}\right) \Phi\left(y, y^{\prime}\right)+\right. \\
& \left.\quad+\Phi^{+}\left(x^{\prime}, y^{\prime}\right) \Phi\left(x, x^{\prime}\right) F\left(y, y^{\prime}\right)\right) \\
(\Omega+\lambda) G(x, y)=- & \frac{\hbar^{2}}{2 m}\left(\Delta_{x}+\Delta_{y}\right) G(x, y)+ \\
& +2 N \iint d x^{\prime} d y^{\prime}\left(V\left(\sqrt[3]{N}\left(x-x^{\prime}\right)\right)+V\left(\sqrt[3]{N}\left(y-y^{\prime}\right)\right)\right) \times \\
& \times\left(F\left(x^{\prime}, y^{\prime}\right) \Phi^{+}\left(x, x^{\prime}\right) \Phi^{+}\left(y, y^{\prime}\right)+\Phi\left(x^{\prime}, y^{\prime}\right) G\left(x, x^{\prime}\right) \Phi^{+}\left(y, y^{\prime}\right)+\right. \\
& \left.\quad+\Phi\left(x^{\prime}, y^{\prime}\right) \Phi^{+}\left(x, x^{\prime}\right) G\left(y, y^{\prime}\right)\right) .
\end{aligned}
$$

Для нахождения спектра квазичастиц из всех решений системы уравнений в вариациях необходимо выделить те, которые удовлетворяют определенному в [20] правилу отбора для комплексного ростка для несамосопряженных операторов с действительным спектром.

Если $k_{2}=0$, то соответствующая этому решению асимптотическая серия является боголюбовской серией, отвечающей скорости течения $\hbar k_{1} / m$. Спектр квазичастиц этой серии выражается известной формулой

$$
\lambda_{l}=\sqrt{\left(\frac{\hbar^{2} l^{2}}{2 m}+V_{0}\right)^{2}-V_{0}^{2}}-\frac{\hbar^{2} l k_{1}}{m} .
$$


Рассмотрим случай $k_{2} \neq 0$. Подставим решения $(35),(36)$ в (46) и учтем симметрию. Тогда получим, что решения системы уравнений в вариациях имеют вид

$$
\begin{aligned}
G_{l}(x, y)= & u_{1, l}\left(e^{i\left(-k_{1}+k_{2}\right) x+i\left(-k_{1}+l\right) y}+e^{i\left(-k_{1}+k_{2}\right) y+i\left(-k_{1}+l\right) x}\right)+ \\
& +u_{2, l}\left(e^{i\left(-k_{1}-k_{2}\right) x+i\left(-k_{1}+2 k_{2}+l\right) y}+e^{i\left(-k_{1}-k_{2}\right) y+i\left(-k_{1}+2 k_{2}+l\right) x}\right), \\
F_{l}(x, y)=- & v_{1, l}\left(e^{i\left(k_{1}+k_{2}\right) x+i\left(k_{1}+l\right) y}+e^{i\left(k_{1}+k_{2}\right) y+i\left(k_{1}+l\right) x}\right)- \\
& -v_{2, l}\left(e^{i\left(k_{1}-k_{2}\right) x+i\left(k_{1}+2 k_{2}+l\right) y}+e^{i\left(k_{1}-k_{2}\right) y+i\left(k_{1}+2 k_{2}+l\right) x}\right)+ \\
& +\sum_{l^{\prime} \neq l, l+2 k_{2}} w_{l, l^{\prime}}\left(e^{i\left(k_{1}+k_{2}+l-l^{\prime}\right) x+i\left(k_{1}+l^{\prime}\right) y}+e^{i\left(k_{1}+k_{2}+l-l^{\prime}\right) y+i\left(k_{1}+l^{\prime}\right) x}\right),
\end{aligned}
$$

где $l \neq-k_{2}$, а числовые коэффициенты $u_{1, l}, u_{2, l}, v_{1, l}, v_{2, l}, w_{l, l^{\prime}}$ определяются из бесконечной системы уравнений. Эта система уравнений содержит замкнутую подсистему из четырех уравнений для коэффициентов $u_{1, l}, u_{2, l}, v_{1, l}, v_{2, l}$, которая может быть записана в стандартном виде

$$
\tilde{\lambda} X=M X
$$

где

$$
\begin{aligned}
& \widetilde{\lambda}=\lambda+\frac{\hbar^{2}}{m} k_{1}\left(k_{2}+l\right), \quad X=\left(\begin{array}{c}
u_{1, l} \\
u_{2, l} \\
v_{1, l} \\
v_{2, l}
\end{array}\right), \\
& M=\left(\begin{array}{cccc}
B_{l} & V_{0} & -V_{0} & 0 \\
V_{0} & B_{l+2 k_{2}} & 0 & -V_{0} \\
4 V_{0} \varphi_{k_{2}, l} & 2 V_{0}\left(\varphi_{k_{2}, l}+\varphi_{k_{2}, l+2 k_{2}}\right) & -B_{l} & -V_{0} \\
2 V_{0}\left(\varphi_{k_{2}, l}+\varphi_{k_{2}, l+2 k_{2}}\right) & 4 V_{0} \varphi_{k_{2}, l+2 k_{2}} & -V_{0} & -B_{l+2 k_{2}}
\end{array}\right), \\
& B_{l}=\frac{\hbar^{2}}{2 m}\left(l^{2}-k_{2}^{2}\right)+2 V_{0} \varphi_{k_{2}, l} .
\end{aligned}
$$

Матрицу $M$ можно представить в виде блочной матрицы

$$
M=\left(\begin{array}{cc}
C & -V_{0} E \\
D & -C
\end{array}\right)
$$

состоящей из $(2 \times 2)$-матриц

$$
C=\left(\begin{array}{cc}
B_{l} & V_{0} \\
V_{0} & B_{l+2 k_{2}}
\end{array}\right), \quad D=\left(\begin{array}{cc}
4 V_{0} \varphi_{k_{2}, l} & 2 V_{0}\left(\varphi_{k_{2}, l}+\varphi_{k_{2}, l+2 k_{2}}\right) \\
2 V_{0}\left(\varphi_{k_{2}, l}+\varphi_{k_{2}, l+2 k_{2}}\right) & 4 V_{0} \varphi_{k_{2}, l+2 k_{2}}
\end{array}\right)
$$

и единичной матрицы $E$.

Такое представление позволяет записать уравнение (49) в виде

$$
\tilde{\lambda}^{2} u=\left(C^{2}-V_{0} D\right) u
$$

для вектор-столбца $u=\left(\begin{array}{l}u_{1, l} \\ u_{2, l}\end{array}\right)$. 
Правила отбора для решений (48) системы уравнений в вариациях [1] выделяют первую серию собственных значений из нижеприведенных:

$$
\begin{aligned}
& \lambda_{1, k_{1}, k_{2}, l}=-\frac{\hbar^{2}}{m} k_{1}\left(k_{2}+l\right)+ \\
&+\frac{\hbar^{2}}{2 m} \sqrt{k_{2}^{4}+\frac{l^{4}}{2}+\frac{l_{1}^{4}}{2}-k_{2}^{2}\left(l^{2}+l_{1}^{2}\right)+\frac{1}{2}\left(l^{2}+l_{1}^{2}-2 k_{2}^{2}\right) \sqrt{\left(l^{2}-l_{1}^{2}\right)^{2}+\left(\frac{4 m V_{0}}{\hbar^{2}}\right)^{2}}} \\
& \lambda_{2, k_{1}, k_{2}, l}=-\frac{\hbar^{2}}{m} k_{1}\left(k_{2}+l\right) \pm \\
& \pm \frac{\hbar^{2}}{2 m} \sqrt{k_{2}^{4}+\frac{l^{4}}{2}+\frac{l_{1}^{4}}{2}-k_{2}^{2}\left(l^{2}+l_{1}^{2}\right)-\frac{1}{2}\left(l^{2}+l_{1}^{2}-2 k_{2}^{2}\right) \sqrt{\left(l^{2}-l_{1}^{2}\right)^{2}+\left(\frac{4 m V_{0}}{\hbar^{2}}\right)^{2}}}
\end{aligned}
$$

где $l_{1}=l+2 k_{2}$ и $l \neq k_{2}$.

Формулы (50) при $l \neq-k_{2}$ задают спектр квазичастиц серии, отвечающей решениям (35), (36). Из явного вида (50) после применения правил отбора для комплексного ростка в несамосопряженной ситуации [20] следует, что в спектре квазичастиц присутствуют только положительные значения. Следовательно, серия, соответствующая гладкому решению (35), (36) при $k_{2} \neq 0$, является метастабильной. Будем считать далее, что $L_{1} \gg L_{2}$. Рассмотрим боголюбовскую серию, соответствующую течению вдоль капилляра со скоростью $\hbar k_{0} / m$, где $k_{0}=2 \pi\left(n_{1} / L_{1}, 0,0\right)$. Для рассматриваемой системы бозонов главный член асимптотики собственных значений этой серии равен

$$
N\left(\frac{\hbar^{2} k_{0}^{2}}{2 m}+\frac{V_{0}}{2}\right) .
$$

Допустим теперь, что соотношение между $L_{1}$ и $L_{2}$ таково, что существует пара векторов $k_{1}, k=2 \pi\left(0, n_{2} / L_{2}, n_{3} / L_{2}\right)$ такая, что соответствующее ей значение $(45)$ в точности совпадает с главным членом (51). Это означает, что между сверхтекучими состояниями боголюбовской серии и несверхтекучими состояниями неметастабильной серии, соответствующей ветвящимся решениям [12], возможен резонанс. Если $L_{1}$ очень велико, резонанс также возможен в случае, когда значение серии, отвечающей ветвящимся решениям, близко к (51) и не обязательно точно с ним совпадает. Существование такого резонанса означает возможность перехода из сверхтекучего состояния в неметастабильное, из которого система перейдет на нижний энергетический уровень, что означает потерю сверхтекучести. Минимальная энергия, отвечающая неметастабильной серии, соответствует случаю $k=2 \pi\left(0,1 / L_{2}, 0\right)$ и согласно формуле (45) равна

$$
E_{\min }=N\left(\frac{\hbar^{2}(2 \pi)^{2}}{2 m L_{2}^{2}}+\frac{V_{0}}{2}\right) .
$$

Сравнивая (52) с (51), получаем, что резонанс невозможен, если скорость течения $v$ по модулю меньше некоторой величины:

$$
|v|<v_{\mathrm{c}}\left(L_{2}\right) \equiv \frac{2 \pi \hbar}{m L_{2}}=\frac{h}{m L_{2}},
$$


что отвечает появлению вихревой нити. Величина $v_{\mathrm{c}}\left(L_{2}\right)$ в правой части неравенства (53) увеличивается с уменьшением толщины капилляра $L_{2}$. Если $L_{2}$ меньше величины $2 \pi \hbar /\left(m v_{\mathrm{cL}}\right)$, где $v_{\mathrm{cL}}$ - критическая скорость Ландау, определяемая из (50) (она существенно больше, чем критическая скорость Ландау, определенная из (47)), и в этом случае сверхтекучесть исчезает при достижении скорости Ландау. Однако при увеличении $L_{2}$ резонанс между сверхтекучим и неметастабильным состояниями наступает при скоростях, меньших скорости Ландау, и это объясняет зависимость критической скорости от толщины капилляра.

Поскольку кривая Ландау и квазичастицы Боголюбова, как мы показали в [3], сохраняются в классическом пределе, то все условия остаются справедливыми для классической жидкости, а значит, при условии $v<h /\left(m L_{2}\right)$, где $L_{2}$ - диаметр нанотрубки, имеет место математический факт: классическая жидкость в нанотрубке должна быть сверхтекучей.

При конечных $l$ мы получили серии, которые дают затухание. Однако в формуле Боголюбова большие $l$ играют роль, поэтому мы рассмотрим случай больших $l$. В этом случае $V_{l}$ при $N \rightarrow \infty$ не сходится к $V_{0}$. Следовательно, уравнения (48), (49) приводят к более точной матрице $M$, элементы которой имеют вид

$$
\begin{array}{ll}
M_{11}=B_{l}+\frac{v_{l-k_{2}}}{2}, & M_{12}=M_{21}=\frac{v_{2 k_{2}}+v_{l+k_{2}}}{2}, \\
M_{13}=-\frac{v_{l+k_{2}}+v_{l-k_{2}}}{2}, \quad M_{14}=M_{23}=0, & M_{22}=B_{l+2 k_{2}}+\frac{v_{l+3 k_{2}}}{2}, \\
M_{24}=-\frac{v_{l+k_{2}}+v_{l+3 k_{2}}}{2}, & M_{31}=2\left(v_{0}+v_{l-k_{2}}\right) \varphi_{k_{2}, l}, \\
M_{32}=M_{41}=\left(v_{2 k_{2}}+v_{l+k_{2}}\right)\left(\varphi_{k_{2}, l}+\varphi_{k_{2}, l+2 k_{2}}\right), & M_{33}=-B_{l}-\frac{v_{l-k_{2}}}{2}, \\
M_{34}=M_{43}=-\frac{v_{2 k_{2}}+v_{l+k_{2}}}{2} & M_{42}=2\left(v_{0}+v_{l+3 k_{2}}\right) \varphi_{k_{2}, l+2 k_{2}}, \\
M_{44}=-B_{l+2 k_{2}}-\frac{v_{l+3 k_{2}}}{2}, &
\end{array}
$$

где

$$
B_{l}=\frac{\hbar^{2}}{2 m}\left(l^{2}-k_{2}^{2}\right)+\left(v_{l-k_{2}}+v_{l+k_{2}}\right) \varphi_{k_{2}, l}-\frac{v_{2 k_{2}}}{2} .
$$

Очевидно, что

$$
v_{l+k_{2}}+v_{l+3 k_{2}}=v_{l-k_{2}}+v_{l+k_{2}}+O\left(\frac{1}{N}\right)
$$

равномерно по $l$ при $l \rightarrow \infty$ (достаточно сделать замену типа (39)). Тогда матрицу $M$ приближенно можно представить в виде блочной матрицы

$$
M=\left(\begin{array}{cc}
C & -V_{l} E \\
D & -C
\end{array}\right)
$$

где $E$ - единичная $(2 \times 2)$-матрица, $V_{l}=\left(v_{l-k_{2}}+v_{l+k_{2}}\right) / 2$. Введем также обозначения $V_{l}^{+}=\left(v_{l+k_{2}}+v_{2 k_{2}}\right) / 2, V_{l}^{-}=\left(v_{l-k_{2}}+v_{0}\right) / 2$. Собственные значения, соответствующие 
уравнению (49), после применения правил отбора принимают вид

$$
\begin{aligned}
\lambda_{k_{1}, k_{2}, l} & =-2 a k_{1}\left(k_{2}+l\right)+ \\
& +\left(\frac{1}{2}\left(a\left(l^{2}-k_{2}^{2}\right)+V_{l}-V_{l}^{+}\right)^{2}+\frac{1}{2}\left(a\left(l_{1}^{2}-k_{2}^{2}\right)+V_{l}-V_{l}^{+}\right)^{2}+V_{l}^{+^{2}}-V_{l}^{2}+\right. \\
& \left.+\frac{1}{2}\left(a\left(l_{1}^{2}+l^{2}-2 k_{2}^{2}\right)+2 V_{l}-2 V_{l}^{+}\right) \sqrt{a^{2}\left(l_{1}^{2}-l^{2}\right)^{2}+4 V_{l}^{+2}}\right)^{1 / 2},
\end{aligned}
$$

где $a=\hbar^{2} /(2 m), l_{1}=l+2 k_{2}$. В этом случае при $k_{2}=0$

$$
\begin{gathered}
\lambda_{k_{1}, k_{2}, l}=-2 a k_{1} l+\left(\left(a l^{2}+V_{l}-V_{l}^{+}\right)^{2}+V_{l}^{+^{2}}-V_{l}^{2}+2\left(a l^{2}+V_{l}-V_{l}^{+}\right)\left|V_{l}^{+}\right|\right)^{1 / 2} \\
V_{l}=v_{l}, \quad V_{l}^{+}=V_{l}^{-}=\frac{v_{l}+v_{0}}{2}
\end{gathered}
$$

Формально, положив $k_{2}=0$, мы приходим к известной формуле Боголюбова

$$
\lambda_{1, l}=-\frac{\hbar^{2}}{m} k_{1} l+\sqrt{\left(\frac{\hbar^{2} l^{2}}{2 m}+v_{l}\right)^{2}-v_{l}^{2}}
$$

где фурье-образ потенциала $v_{l}$ при $l \gg \sqrt[3]{N}$ стремится к нулю, и тогда основную роль начинает играть кинетический член.

Мы полагаем, что $L_{2}$ много больше некоторой стандартной длины, например радиуса электрона $r_{0}$, и хотя $L_{1} \gg L_{2}$, мы можем выбрать достаточно большое целое число $n$. Иначе говоря, $L_{2} / r_{0} \rightarrow \infty, L_{1} / r_{0} \rightarrow \infty$, но вектор $k_{1}=\left(n / L_{1}, 0,0\right)$ конечен, так как $n \rightarrow \infty$. На языке нестандартного анализа это означает следующее: $L_{2}-$ бесконечно большое (нестандартное) число, $L_{1}$ и $n_{1}$ - нестандартные числа более высокого порядка, а $k_{1}=\left(n / L_{1}, 0,0\right)$ - стандартное конечное число. Тогда $k_{2}$ равно бесконечно малому (нестандартному) нулю: $k_{2} \cong 0$, а $k_{1}$ - стандартное число.

\section{5. СЛУЧАЙ НЕЧЕТНОГО ЧИСЛА НЕЙТРОНОВ}

Рассмотрим систему уравнений Гамильтона для фермионов

$$
\begin{aligned}
& \Omega \Phi(x, y)=-\frac{\hbar^{2}}{2 m}\left(\Delta_{x}+\Delta_{y}\right) \Phi(x, y)+ \\
& +2 N \iint d x^{\prime} d y^{\prime}\left(V(x-y)+V\left(x^{\prime}-y^{\prime}\right)\right) \Phi^{+}\left(x^{\prime}, y^{\prime}\right) \Phi\left(x, x^{\prime}\right) \Phi\left(y^{\prime}, y\right), \\
& \Omega \Phi^{+}(x, y)=-\frac{\hbar^{2}}{2 m}\left(\Delta_{x}+\Delta_{y}\right) \Phi^{+}(x, y)+ \\
& \quad+2 N \iint d x^{\prime} d y^{\prime}\left(V\left(x-x^{\prime}\right)+V\left(y-y^{\prime}\right)\right) \Phi\left(x^{\prime}, y^{\prime}\right) \Phi^{+}\left(x, x^{\prime}\right) \Phi^{+}\left(y^{\prime}, y\right) .
\end{aligned}
$$

Функции $\Phi^{+}(x, y), \Phi(x, y)$ являются антисимметричными и удовлетворяют условию нормировки (32). Представим потенциал взаимодействия в виде ряда Фурье:

$$
N V(x)=\sum_{p} v_{p} e^{i p x}, \quad v_{p}=\frac{1}{L_{1} L_{2}^{2}} \int d x N V(x) e^{-i p x}, \quad v_{p}=v_{-p} .
$$


Решение системы уравнений (57), (32) будем искать в виде

$$
\begin{aligned}
& \Phi_{k_{1}, k_{2}}^{+}(x, y)=\frac{1}{L_{1} L_{2}^{2}} e^{-i k_{1}(x+y)} \sin \left(k_{2}(x-y)\right), \\
& \Phi_{k_{1}, k_{2}}(x, y)=\frac{1}{L_{1} L_{2}^{2}} \sum_{l} \varphi_{k_{2}, l} e^{i l(x-y)+i k_{1}(x+y)},
\end{aligned}
$$

где $k_{1}, k_{2}, l$ - трехмерные векторы вида $2 \pi\left(n_{1} / L_{1}, n_{2} / L_{2}, n_{3} / L_{2}\right), n_{1}, n_{2}, n_{3}$ - целые числа. Числа $\varphi_{k_{2}, l}$ должны удовлетворять условию $\varphi_{k_{2}, l}=-\varphi_{k_{2},-l}$. После подстановки получаем, что собственное значение равно

$$
\Omega=\frac{\hbar^{2}}{m}\left(k_{1}^{2}+k_{2}^{2}\right)+v_{2 k_{2}}-v_{0},
$$

а $\varphi_{k_{2}, l}$ имеют вид

$$
\varphi_{k_{2}, l}=-\frac{i b_{k_{2}, l}}{2} \pm \frac{1}{2} \sqrt{1-b_{k_{2}, l}^{2}}, \quad b_{k_{2}, l} \equiv \frac{\frac{\hbar^{2}}{m}\left(l^{2}-k_{2}^{2}\right)+v_{0}-v_{2 k_{2}}}{v_{l-k_{2}}-v_{l+k_{2}}} .
$$

Отметим, что $b_{k_{2}, l}=-b_{k_{2},-l}$. Положим

$$
\varphi_{k_{2}, l}=-\frac{i b_{k_{2}, l}}{2}+\frac{1}{2} \frac{v_{l-k_{2}}-v_{l+k_{2}}}{\left|v_{l-k_{2}}-v_{l+k_{2}}\right|} \sqrt{1-b_{k_{2}, l}^{2}} .
$$

Тогда $\varphi_{k_{2}, l}$ будет равно $-\varphi_{k_{2},-l}$.

Рассмотрим систему уравнений в вариациях для фермионов

$$
\begin{aligned}
& (\Omega-\lambda) F(x, y)=-\frac{\hbar^{2}}{2 m}\left(\Delta_{x}+\Delta_{y}\right) F(x, y)+ \\
& \quad+2 N \iint d x^{\prime} d y^{\prime}\left(V(\sqrt[3]{N}(x-y))+V\left(\sqrt[3]{N}\left(x^{\prime}-y^{\prime}\right)\right)\right) \times \\
& \quad \times r\left(G\left(x^{\prime}, y^{\prime}\right) \Phi\left(x, x^{\prime}\right) \Phi\left(y^{\prime}, y\right)+\Phi^{+}\left(x^{\prime}, y^{\prime}\right) F\left(x, x^{\prime}\right) \Phi\left(y^{\prime}, y\right)+\right. \\
& \left.\quad+\Phi^{+}\left(x^{\prime}, y^{\prime}\right) \Phi\left(x, x^{\prime}\right) F\left(y^{\prime}, y\right)\right), \\
& (\Omega+\lambda) G(x, y)=-\frac{\hbar^{2}}{2 m}\left(\Delta_{x}+\Delta_{y}\right) G(x, y)+ \\
& \quad+2 N \iint d x^{\prime} d y^{\prime}\left(V\left(\sqrt[3]{N}\left(x-x^{\prime}\right)\right)+V\left(\sqrt[3]{N}\left(y-y^{\prime}\right)\right)\right) \times \\
& \quad \times\left(F\left(x^{\prime}, y^{\prime}\right) \Phi^{+}\left(x, x^{\prime}\right) \Phi^{+}\left(y^{\prime}, y\right)+\Phi\left(x^{\prime}, y^{\prime}\right) G\left(x, x^{\prime}\right) \Phi^{+}\left(y^{\prime}, y\right)+\right. \\
& \left.\quad+\Phi\left(x^{\prime}, y^{\prime}\right) \Phi^{+}\left(x, x^{\prime}\right) G\left(y^{\prime}, y\right)\right) .
\end{aligned}
$$

Решения системы уравнений в вариациях имеют вид

$$
\begin{aligned}
G_{l}(x, y)= & u_{1, l}\left(e^{i\left(-k_{1}+k_{2}\right) x+i\left(-k_{1}+l\right) y}-e^{i\left(-k_{1}+k_{2}\right) y+i\left(-k_{1}+l\right) x}\right)+ \\
& +u_{2, l}\left(e^{i\left(-k_{1}-k_{2}\right) x+i\left(-k_{1}+2 k_{2}+l\right) y}-e^{i\left(-k_{1}-k_{2}\right) y+i\left(-k_{1}+2 k_{2}+l\right) x}\right), \\
F_{l}(x, y)=v_{1, l}\left(e^{i\left(k_{1}+k_{2}\right) x+i\left(k_{1}+l\right) y}-e^{i\left(k_{1}+k_{2}\right) y+i\left(k_{1}+l\right) x}\right)+ & +v_{2, l}\left(e^{i\left(k_{1}-k_{2}\right) x+i\left(k_{1}+2 k_{2}+l\right) y}-e^{i\left(k_{1}-k_{2}\right) y+i\left(k_{1}+2 k_{2}+l\right) x}\right)+ \\
& +\sum_{l^{\prime} \neq l, l+2 k_{2}} w_{l, l^{\prime}}\left(e^{i\left(k_{1}+k_{2}+l-l^{\prime}\right) x+i\left(k_{1}+l^{\prime}\right) y}-e^{i\left(k_{1}+k_{2}+l-l^{\prime}\right) y+i\left(k_{1}+l^{\prime}\right) x}\right),
\end{aligned}
$$


где $l \neq-k_{2}$, а числовые коэффициенты $u_{1, l}, u_{2, l}, v_{1, l}, v_{2, l}, w_{l, l^{\prime}}$ определяются из бесконечной системы уравнений. Эта система уравнений содержит замкнутую подсистему из четырех уравнений для коэффициентов $u_{1, l}, u_{2, l}, v_{1, l}, v_{2, l}$, которая может быть записана в стандартном виде (49).

Матрица $M$ имеет элементы

$$
\begin{array}{ll}
M_{11}=B_{l}+\frac{v_{l-k_{2}}}{2}, & M_{12}=M_{21}=\frac{v_{l+k_{2}}-v_{2 k_{2}}}{2}, \\
M_{13}=\frac{v_{l-k_{2}}-v_{l+k_{2}}}{2}, & M_{14}=M_{23}=0, \\
M_{22}=B_{l+2 k_{2}}+\frac{v_{l+3 k_{2}}}{2}, & M_{24}=\frac{v_{l+3 k_{2}}-v_{l+k_{2}}}{2}, \\
M_{31}=2 i\left(v_{l-k_{2}}-v_{0}\right) \varphi_{k_{2}, l}, & M_{32}=M_{41}=i\left(v_{2 k_{2}}-v_{l+k_{2}}\right)\left(\varphi_{k_{2}, l+2 k_{2}}-\varphi_{k_{2}, l}\right), \\
M_{33}=-B_{l}-\frac{v_{l-k_{2}}}{2}, & M_{34}=M_{43}=-\frac{v_{l+k_{2}}-v_{2 k_{2}}}{2}, \\
M_{42}=2 i\left(v_{0}-v_{l+3 k_{2}}\right) \varphi_{k_{2}, l+2 k_{2}}, & M_{44}=-B_{l+2 k_{2}}-\frac{v_{l+3 k_{2}}}{2},
\end{array}
$$

где

$$
B_{l}=\frac{\hbar^{2}}{2 m}\left(l^{2}-k_{2}^{2}\right)+i\left(v_{l+k_{2}}-v_{l-k_{2}}\right) \varphi_{k_{2}, l}-\frac{v_{2 k_{2}}}{2} .
$$

Собственные значения, соответствующие уравнению (49), при $k_{2} \neq 0$ также содержат после применения правил отбора положительные квазичастицы.

При $k_{2}=0$ собственные значения имеют вид

$$
\lambda_{1, l}=-\frac{\hbar^{2}}{m} l k_{1} \pm \frac{\hbar^{2} l^{2}}{2 m}, \quad \lambda_{2, l}=-\frac{\hbar^{2}}{m} l k_{1} \pm\left|\frac{\hbar^{2} l^{2}}{2 m}+v_{l}-v_{0}\right| .
$$

Это значит, что мы выбираем векторы $k_{2}=\left(0,1 / L_{2}, 0\right), k_{1}=\left(n / L_{1}, 0,0\right)$. Если $n \gg 1$, а следовательно, $k_{1} \gg k_{2}$, это значит, что вектор скорости жидкости в основном направлен вдоль трубки. Пусть, как и ранее, $L_{1}=\infty, L_{2}=\infty, n=\infty-$ нестандартные числа, $L_{1} \gg L_{2}, k_{1}$ - стандартное число. Тогда $k_{2} \cong 0, \varphi_{k_{2}, l}$ принимает при $k_{2} \cong 0$ нестандартные значения. Однако если $0 \leqslant l \leqslant \sqrt{k_{2}} M$, где $M \leqslant \infty$, т.е. $0 \leqslant l \leqslant \infty$, то $\varphi_{k_{2}, l}$ можно считать стандартным числом. При $k_{2} \cong 0$, учитывая правило отбора, мы получаем аналогично формуле (56) в бозонном случае

$$
\lambda_{2, l}=-\frac{\hbar^{2}}{m} l k_{1}+\left|\frac{\hbar^{2} l^{2}}{2 m}+v_{l}-v_{0}\right| .
$$

При $|l| \rightarrow 0$ в силу п. 1.2

$$
v_{l}-v_{0} \sim-4 \pi|l| \lim _{r \rightarrow \infty} r^{4} V(r) .
$$

а так как $V(r)$ при $r \rightarrow \infty-$ притягивающий потенциал, то

$$
-4 \pi \lim _{r \rightarrow \infty} r^{4} V(r)=c>0 .
$$


При $l \rightarrow \infty$ выражение под знаком модуля стремится к бесконечности как $\hbar^{2} l^{2} /(2 m)$, так как $v_{l} \rightarrow \infty$ как $1 /|l|^{2}$. Таким образом, поведение выражения под знаком модуля в (62) аналогично изменению кривой Ландау для бозонов. Критерий для $k_{1}$ имеет вид

$$
\left|k_{1}\right| \leqslant \frac{m}{\hbar^{2}} \min _{l}\left|\frac{v_{l}-v_{0}}{|l|}+\frac{\hbar^{2}|l|}{2 m}\right|,
$$

аналогично критерию Ландау. Разумеется, ограничение (53) здесь также имеет место.

Таким образом, мы видим, что если для возникновения сверхтекучести бозонов важно отталкивание на близких расстояниях, то в сверхтекучести фермионов основную роль играет притяжение на дальних расстояниях. И в том, и в другом случае переход в конденсатное состояние (для пар в ферми-жидкости) является фазовым переходом, не имеющим отношения к сверхтекучести.

Зависимость от температуры будет изучена в другой работе.

ЗАмЕчАниЕ. Ограничение снизу для толщины нанотрубки определяется величиной дальнодействия потенциала взаимодействия.

Благодарности. Автор выражает благодарность Д. С. Голикову за пересчет и проверку всех формул.

\section{Список литературы}

[1] В. П. Маслов, Комплексный метод ВКБ в нелинейных уравнениях, Наука, М., 1977.

[2] Н.Н. Боголюбов, "К теории сверхтекучести", Избранные труды в трех томах, т. 2, Наукова думка, Киев, 1970, 210-224.

[3] V.P. Maslov, Russ. J. Math. Phys., 3:3 (1995), 401-406.

[4] В. П. Маслов, О. Ю. Шведов, Метод комплексного ростка в многочастичных задачах и задачах квантовой теории поля, УРСС, М., 2000.

[5] Л. Д. Ландау, "К теории сверхтекучести”, Собрание трудов, т. 2, Наука, М., 1969, 42-46; L. D. Landau, Phys. Rev., 75:5 (1949), 884-885.

[6] В.П. Маслов, Квантование термодинамики и ультравторичное квантование, Ин-т компьютерных исследований, М., 2001.

[7] В. П. Маслов, Функи. анализ и его прилож., 34:4 (2000), 35-48.

[8] В. П. Маслов, УМН, 55:6 (2000), 145-146.

[9] V.P. Maslov, Russ. J. Math. Phys., 8:3 (2001), 309-321.

[10] V.P. Maslov, Russ. J. Math. Phys., 8:1 (2001), 55-82.

[11] В. П. Маслов, ТМФ, 132:3 (2002), 388-398.

[12] Е. М. Лифшиц, Л. П. Питаевский, Статистическая физика. Ч. 2. Теория конденсированного состояния, Наука, М., 1978.

[13] В. П. Маслов, ТМФ, 143:3 (2005), 307-327.

[14] V.P. Maslov, Russ. J. Math. Phys., 12:3 (2005), 369-378.

[15] В. П. Маслов, ДАН СССР, 151:2 (1963), 306-309.

[16] В. П. Маслов, ТМФ, 131:2 (2002), 261-277.

[17] В. П. Маслов, ТМФ, 125:2 (2000), 297-314.

[18] В. П. Маслов, ТМФ, 129:3 (2001), 464-490.

[19] Ф.А. Березин, Метод вторичного квантования, Наука, М., 1986.

[20] В. П. Маслов, Операторные методы, Наука, М., 1973. 